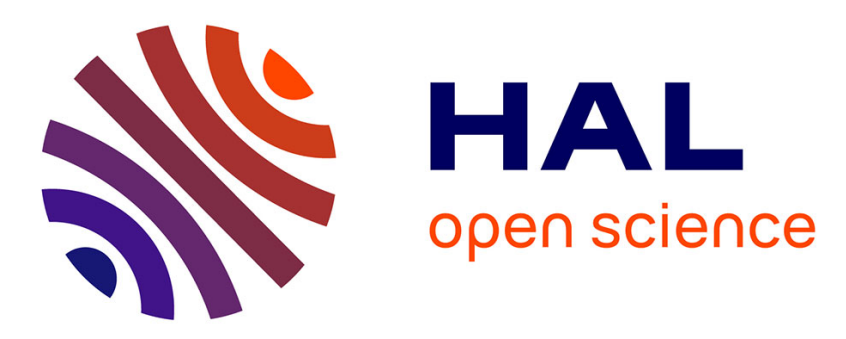

\title{
Inherently gain flattened L+ Band TDFA based on W-fiber Design
}

Charu Kakkar, Gérard Monnom, Krishna Thyagarajan, Bernard Dussardier

\section{To cite this version:}

Charu Kakkar, Gérard Monnom, Krishna Thyagarajan, Bernard Dussardier. Inherently gain flattened L+ Band TDFA based on W-fiber Design. Optics Communications, 2006, 262 (2), pp.193-199. 10.1016/j.optcom.2005.12.059 . hal-00508607

\section{HAL Id: hal-00508607 https://hal.science/hal-00508607}

Submitted on 5 Aug 2010

HAL is a multi-disciplinary open access archive for the deposit and dissemination of scientific research documents, whether they are published or not. The documents may come from teaching and research institutions in France or abroad, or from public or private research centers.
L'archive ouverte pluridisciplinaire HAL, est destinée au dépôt et à la diffusion de documents scientifiques de niveau recherche, publiés ou non, émanant des établissements d'enseignement et de recherche français ou étrangers, des laboratoires publics ou privés. 


\title{
Inherently gain flattened $\mathrm{L}+$ Band TDFA based on $\boldsymbol{W}$-fiber Design
}

\author{
Charu Kakkar $^{1,2}$, Gérard Monnom ${ }^{1}$, K. Thyagarajan ${ }^{2}$, Bernard Dussardier ${ }^{1}$ \\ ${ }^{1}$ Laboratoire de Physique de la Matière Condensée CNRS, Université de Nice-Sophia Antipolis, Parc Valrose, \\ 06108 Nice, France \\ ${ }^{2}$ Department of Physics, Indian Institute of Technology Delhi, Hauz Khas, New Delhi, 110016, India
}

Abstract: We present here a detailed theoretical analysis for realising an inherently gain flattened $L+$ band Thulium doped fiber amplifier (TDFA), based on a depressed inner-clad (W-fiber) design, wherein the inherent gain flattening is achieved by an optimised bend induced leakage loss. The leakage loss characteristics of $W$-fiber have been used to suppress higher wavelength amplified stimulated emission (ASE) in the designed TDFA, which otherwise depletes the population inversion in the amplifier, making it almost impossible to obtain high gain for wavelengths in and close to the conventional L-band. It has been shown through simulations that $20 \mathrm{~dB}$ net gain ( $\pm 0.3 \mathrm{~dB}$ ripple) is achievable over $32 \mathrm{~nm}$ bandwidth (1604-1636 nm), using this design pumped with $160 \mathrm{~mW}$ of power. We also show that inherent gain flattening leads to redistribution of power among signal wavelengths, and hence an inherently gain-flattened TDFA is much more efficient as compared to a configuration that uses discrete filters for gain flattening. The net gain value and gain flattening of designed TDFA module has been tested against tolerance w.r.t. fiber parameters as well as bend radius. This is for the first time to the authors knowledge that inherently gain flattened L+ band operation has been shown using TDFAs.

Keywords: Thulium, Leaky mode, L-band, Gain flattening, Optical amplifier, Leakage loss 


\section{Introduction}

Extending optical fiber transmission to newer wavelength bands (S-band 1470-1525 $\mathrm{nm}$, L-band $\sim 1565-1620 \mathrm{~nm}$, O-band $\sim 1260-1310 \mathrm{~nm}$ ) is a potential solution for increasing the capacity of current wavelength division multiplexed (WDM) and coarse wavelength division multiplexed (CWDM) systems; and gain-flattened optical fiber amplifiers are key devices in this direction. There has been a lot of activity in realising efficient L-band Erbium doped fiber amplifiers (EDFAs) $[1,2]$ and also in extending the amplifier bandwidth beyond conventional L-band. However, in conventional L-EDFAs, the bandwidth of operation is restricted till about $1610 \mathrm{~nm}$ only and thus, a number of techniques have been reported in the literature to extend the bandwidth of L-band amplifiers. Some of these include the use of antimony silicate glass host, instead of $\mathrm{Al} / \mathrm{Si}$ [3] or the use of Raman amplification [4].

In this paper, we report an inherently gain flattened, fluoride based thulium doped fiber amplifier (TDFA) with $20 \mathrm{~dB}$ net gain $( \pm 0.3 \mathrm{~dB}$ ripple) over $32 \mathrm{~nm}$ wide bandwidth from $1604 \mathrm{~nm}$ to $1636 \mathrm{~nm}$. Thulium $\left(\mathrm{Tm}^{3+}\right)$ is an important rare earth element which has been predominantly used for realising S-band amplifiers [5] based on ${ }^{3} \mathrm{H}_{4}{ }^{3} \mathrm{~F}_{4}$ transition peaked around $1.47 \mu \mathrm{m}$, and long wavelength lasers [6] based on ${ }^{3} \mathrm{~F}_{4}{ }^{3} \mathrm{H}_{6}$ transition peaked around 1.9 $\mu \mathrm{m}$ (Figure 1). The ${ }^{3} \mathrm{~F}_{4}{ }^{3} \mathrm{H}_{6}$ transition, however, has an emission tail till $1600 \mathrm{~nm}$, and thus in principle, this transition can be used for realising $\mathrm{L}+$ band $(1600-1640 \mathrm{~nm})$ fiber amplifiers. However, efficient L-band TDFAs cannot be realised using conventional fiber designs because the higher wavelength amplified spontaneous emission (ASE) would deplete the population inversion in the amplifier, making it virtually impossible to amplify L band signals. Thus, to ensure gain at the shorter edge of wavelengths, one must suppress the higher wavelength ASE periodically along the length of the fiber. Sakamoto et al [7] have previously reported a ZBLYAN based TDFA operating over 1650-1670 nm, where higher wavelength (1750-2000 nm) ASE has been suppressed by co-doping the fiber with Terbium $\left(\mathrm{Tb}^{3+}\right)$ ions, 
which have a large absorption cross-section for wavelengths $>1750 \mathrm{~nm}$. The problem with this technique is that the usable amplifier bandwidth in this case is $1650-1670 \mathrm{~nm}$, and the WDM bandwidth between 1600-1650 nm (for which silica based transmission fibers have lower losses) could not be exploited.

In this paper, we propose suppression of higher wavelength ASE in an L-TDFA by an appropriate leakage loss to the fundamental mode, in an optimised $W$-fiber. The fundamental mode in the proposed $W$-fiber becomes leaky at around $1661 \mathrm{~nm}$, so that all the higher wavelength ASE is suppressed, and the gain in L+ band (1600-1640) nm is enhanced. A similar technique of higher wavelength suppression using leakage loss was reported by Arbore et al [8] to realize an S-band EDFA, where C-band ASE is suppressed using leakage loss in a $W$-fiber. This technique has also been applied to filter out strong four level ASE transition and enhance three level transition in Neodymium and Ytterbium doped fibers [9]. We show, using simulations, that even though ASE suppression in a $W$-fiber leads to an increase in L+ band gain as compared to the case of an ordinary step index fiber design, this gain has a large spectral variation, with the gain at $1600 \mathrm{~nm}$ being around $20 \mathrm{~dB}$ lower than that at $1640 \mathrm{~nm}$. To compensate for this gain tilt, use of an optimised wavelength-dependent bend induced leakage loss is proposed. Thus, in the proposed module, a high leakage loss $(>3$ $\mathrm{dB} / \mathrm{m}$ ) is provided to suppress the longer wavelengths, outside the signal band and a spectrally tuned bend induced leakage loss ( 0 to 1 ) $\mathrm{dB} / \mathrm{m}$ is provided to the signal band wavelengths to compensate for the gain tilt. We show that gain flatness upto $\pm 0.3 \mathrm{~dB}$, over $32 \mathrm{~nm}$ bandwidth is achievable in the proposed $\mathrm{L}+$ band TDFA, based on optimally bent $W$-fiber. It is also emphasised, through detailed simulations, that inherent gain flattening is achieved by suppression of gain at higher signal wavelengths and a simultaneous increase in gain at shorter signal wavelengths by a re-distribution of power among the signal channels. This is in contrast to gain flattening by discrete filters, which is achieved by a suppression of gain at all 
the wavelengths. Hence, inherent gain flattening is more efficient than flattening by the use of discrete filters. The gain and gain flatness in the proposed module are also tested against tolerance with respect to fiber parameters and bend radius. In these simulations, we have considered fluoride hosts for realising L+ TDFA; this is essential because the fluorescence lifetime of the upper lasing level $\left({ }^{3} \mathrm{~F}_{4}\right)$ in silica glass is much lower $(\sim 0.4 \mathrm{~ms})$ than in ZBLYAN glass ( $\sim 9 \mathrm{~ms})$, which makes it almost impossible to realise high gain L + TDFA in silica hosts.

\section{Fiber design and basic principle}

The solid curve in Figure 2 shows the refractive index profile of a $W$-fiber, which consists of a depressed inner cladding. This profile can be characterized by a specific wavelength $\lambda_{c}$, defined as follows: at $\lambda_{c}$, the effective index of the fundamental mode equals the outer cladding refractive index $\left(n_{3}\right)$. Thus at $\lambda_{c}$, the guided mode gets cut-off and it starts to behave like a leaky mode, the leakage loss spectrum of which is governed by the separation $b$ - $a$. Hence, by optimising the fiber parameters, one can have different spectral variation of leakage loss for the wavelength range $\lambda>\lambda_{c}$.

The dashed curve in Figure 2 shows the equivalent refractive index profile of a bent $W$ fiber [10]. Bending the fiber, shifts the cut-off wavelength $\lambda_{c}$ to shorter wavelengths, and hence those wavelengths also start to suffer some leakage loss, the amount of which, can be controlled by varying the bend radius. The shorter is the bend radius, the more is the blueshifting of $\lambda_{c}$ and higher are the losses at a particular wavelength. The amount of bendsensitivity of the fiber design can be easily controlled by the fiber parameters, predominantly by the $b / a$ ratio. This implies that to achieve similar bend losses, fibers with larger $b / a$ ratio would require smaller bend radii than fibers with smaller $b / a$ ratio. Bend induced leakage loss has an almost linear spectral variation in $W$-fibers, the slope of which can be tuned by varying 
the bend radius. Hence, an optimally chosen bend radius can flatten the almost linear gain-tilt in an L+ band TDFA, effectively. This is the basic principle behind the proposed inherently gain-flattened L+ band TDFA.

The advantage of having two mechanisms for inducing leakage loss (bend-induced leakage loss and inherent leakage loss in a straight $W$-profile) is that it enables us to control the magnitude and spectral variation of leakage loss inside and outside the signal band, almost independent of each other. This is because leakage loss induced by bend has a different spectral variation than that of the leakage loss in the straight fiber. This gives additional degree of freedom to achieve efficient gain flattening.

\section{Modelling}

In order to analyze amplification in the proposed fiber, we have used standard three-level rate equation model [11], as applicable in the case of an EDFA. $1210 \mathrm{~nm}$ pump has been considered for pumping the atoms into ${ }^{3} \mathrm{H}_{5}$ level, from which, the atoms decay non-radiatively into the upper lasing level $\left({ }^{3} \mathrm{~F}_{4}\right)$ and thus the system acts as a three-level system. One could also utilize $800 \mathrm{~nm}$ band pumping for realizing populations inversion between ${ }^{3} \mathrm{~F}_{4}$ and ${ }^{3} \mathrm{H}_{6}$ levels, but the efficiency of $1210 \mathrm{~nm}$ pumping scheme is higher as compared to $800 \mathrm{~nm}$ pumping. Assuming a cylindrical co-ordinate system with $z$-axis as the fiber axis, the per unit volume steady state population density of $\mathrm{Tm}^{3+}$ ions, in the ground and excited states, $\left(N_{l}(r\right.$, $z$ ) and $\left.N_{2}(r, z)\right)$, are described by the following equations:

$$
\begin{aligned}
& N_{2}(r, z)=\rho_{\mathrm{Er}} \frac{W_{\mathrm{a}}(r, z)}{W_{\mathrm{a}}(r, z)+W_{\mathrm{e}}(r, z)} \\
& N_{1}(r, z)=\rho_{\mathrm{Er}}-N_{2}(r, z)
\end{aligned}
$$


Here, $\rho_{\mathrm{Er}}$ is the total thulium ion concentration, and $\mathrm{W}_{\mathrm{a}}, \mathrm{W}_{\mathrm{e}}$ represent the net emission and absorption rates, given by:

$$
\begin{aligned}
W_{\mathrm{a}}(r, z)= & {\left[\frac{\sigma_{\mathrm{a}}\left(v_{\mathrm{s}}\right)}{h v_{\mathrm{s}}} P_{\mathrm{s}}(z)+\int_{0}^{\infty} \frac{\sigma_{\mathrm{a}}(v)}{h v} S_{\mathrm{ase}}(v, z) \mathrm{d} v\right] I_{\mathrm{s}}(r) } \\
& +\left[\frac{\sigma_{\mathrm{a}}\left(v_{\mathrm{p}}\right)}{h v_{\mathrm{p}}} P_{\mathrm{p}}(z)\right] I_{\mathrm{p}}(r) \\
W_{\mathrm{e}}(r, z)= & {\left[\frac{\sigma_{\mathrm{e}}\left(v_{\mathrm{s}}\right)}{h v_{\mathrm{s}}} P_{\mathrm{s}}(z)+\int_{0}^{\infty} \frac{\sigma_{\mathrm{e}}(v)}{h v} S_{\mathrm{ase}}(v, z) \mathrm{d} v\right] I_{\mathrm{s}}(r)+A_{\mathrm{e}} }
\end{aligned}
$$

Here, $v_{\mathrm{p}, \mathrm{s}}$ is the pump/signal frequency, $\sigma_{\mathrm{a}, \mathrm{s}}$ is the wavelength dependent absorption/emission cross-section and $P_{\mathrm{p}, \mathrm{s}}(\mathrm{r}, \mathrm{z})$ is the pump/signal power at any position $z$, along the fiber length. $A_{\mathrm{e}}$ is the spontaneous emission rate, $S_{\text {ase }}(v, z)$ is the amplified spontaneous emission (ASE) power spectral density at frequency $v$ and position $z$ and, $I_{s}(r)$ and $I_{p}(r)$ are the normalized intensity distributions of the fundamental mode, at signal and pump wavelengths, respectively. Since, the spontaneous emission generated at any point is amplified in both forward and backward directions of the fiber axis, the total ASE spectral density, $\left(S_{\text {ase }}(v, z)\right)$, at any point $z$, can be written as a sum of forward $\left(S^{+}\right.$ase $)$and backward $\left(S_{\text {ase }}\right)$ components.

$$
S_{\text {ase }}(v, z)=S_{\text {ase }}^{+}(v, z)+S_{\text {ase }}^{-}(v, z)
$$

The propagation equations for the pump, signal and ASE are given as follows:

$$
\begin{aligned}
& \frac{\mathrm{d} P_{\mathrm{p}, \mathrm{s}}\left(v_{\mathrm{p}, \mathrm{s}}, z\right)}{\mathrm{d} z}=\left[\gamma_{\mathrm{e}}\left(v_{\mathrm{p}, \mathrm{s}}, z\right)-\gamma_{\mathrm{a}}\left(v_{\mathrm{p}, \mathrm{s}}, z\right)\right] P_{\mathrm{p}, \mathrm{s}}\left(v_{\mathrm{p}, \mathrm{s}}, z\right) \\
& \frac{\mathrm{d} S_{\text {ase }}^{ \pm}(v, z)}{\mathrm{d} z}= \pm 2 h v \gamma_{\mathrm{e}}(v, z) \pm\left[\gamma_{\mathrm{e}}(v, z)-\gamma_{\mathrm{a}}(v, z)\right] S_{\text {ase }}^{ \pm}(v, z)
\end{aligned}
$$


The emission $\gamma_{e}(v, z)$ and absorption $\gamma_{a}(v, z)$ factors in these equations are determined as follows:

$$
\begin{aligned}
& \gamma_{\mathrm{e}}(v, z)=\sigma_{\mathrm{e}}(v) 2 \pi \int_{0}^{a} N_{2}(r, z) I(v, r) r \mathrm{~d} r \\
& \gamma_{\mathrm{a}}(v, z)=\sigma_{\mathrm{a}}(v) 2 \pi \int_{0}^{a} N_{1}(r, z) I(v, r) r \mathrm{~d} r
\end{aligned}
$$

where $a$ is the core radius of the fiber and $I(v, r)$ is the normalized intensity at frequency $v$ and radial co-ordinate $r$.

Effective refractive indices of the fundamental and leaky modes of $W$-fiber and the leakage loss have been obtained by solving complex eigenvalue equation using matrix method [12]. Bend induced leakage loss have been obtained using an analytical analysis given by Marcuse [10]. To obtain the overlap integrals, we have assumed the modal fields of the segmented-clad fiber to be the same as that of the corresponding unperturbed fiber. Modal fields for the unperturbed fiber have been calculated by writing the solutions in each region in terms of Bessel functions and applying appropriate boundary conditions to obtain an eigenvalue equation, which has been solved numerically.

\section{Numerical Results and Discussion}

The refractive indexes of the designed $W$ - fiber have been chosen in a manner that $\lambda_{c}=$ $1661 \mathrm{~nm}$; the signal band is over $1604 \mathrm{~nm}$ to $1636 \mathrm{~nm}$. Parameters of the core $\left(n_{1}, n_{2}, a\right)$ primarily decide the virtual cut-off wavelength $\left(\lambda_{c}\right)$ for the fundamental mode. As mentioned before, the amount of leakage loss in a $W$-fiber, and its sensitivity towards bend loss depends upon $b / a$ ratio. Hence, the fiber dimensions are so chosen that the leakage loss even in a straight $W$-fiber is sufficiently high $(>2.5 \mathrm{~dB} / \mathrm{m})$ to eliminate higher wavelength ASE and also, the fiber is reasonably sensitive to bend induced leakage losses so that very small bend 
radii are not required for obtaining desired leakage loss $(0-1 \mathrm{~dB} / \mathrm{m})$ in the signal band. Figure 3 shows the leakage loss spectrum in the presence and absence of bend, in the designed fiber. The optimised bend radius in this case is $8 \mathrm{~cm}$. The presence of a bend shifts $\lambda_{c}$ to 1604, from its original value at $1661 \mathrm{~nm}$ in the straight fiber.

The designed L+ band TDFA consists of 32 meters of optimized $W$-fiber, pumped with $160 \mathrm{~mW}$ of power at $1210 \mathrm{~nm}$. The core region $(r<a)$ is considered to be doped with thulium with a concentration of $1.7 \times 10^{25} \mathrm{~m}^{-3}$. The propagation equations for pump, signals and forward and backward ASE (Eqn. 6 and 7) have been solved as boundary value problem using fourth order Runge Kutta method. For ASE calculation, $150 \mathrm{~nm}$ emission bandwidth, (1550 to 1700) $\mathrm{nm}$, has been divided into 150 wavelength slots of $1 \mathrm{~nm}$ width each. Due to very large leakage loss $(>8 \mathrm{~dB} / \mathrm{m})$ for wavelengths higher than $1700 \mathrm{~nm}$, the emission band between $1700 \mathrm{~nm}$ to $2200 \mathrm{~nm}$ is not included in the simulations.

Figure 4 shows the small signal gain and noise figure spectra of the designed $\mathrm{L}+$ band TDFA; nine equally spaced simultaneous signal channels over 1604-1636 nm have been considered with a total signal input power of $-10 \mathrm{dBm}$. The figure shows that $\sim 20 \mathrm{~dB}$ net gain with a gain ripple of $\pm 0.3 \mathrm{~dB}$ is achievable with this design. The noise figure remains below 7 $\mathrm{dB}$ for all signal wavelengths. To emphasize the significance of an optimum spectral variation of leakage loss leading to inherent gain flattening, we simulated the performance of an L+ band TDFA for three cases: 1) in a conventional step index fiber with no leakage loss at all, 2) in a straight $W$-fiber, where the higher wavelength ASE were suppressed by a suitably chosen cutoff for fundamental mode, but no gain flattening, 3) in an optimally bent $W$-fiber with suppression of higher wavelength ASE alongwith inherent gain flattening provided by suitably tuned spectral variation of leakage loss in signal band. Figure 5 shows the results for all the three cases. The pump and signal powers and the ion concentration are the same as in Figure 4 for all the three cases; fiber lengths are however optimized for each case to obtain 
maximum gain. One may note that $\mathrm{L}+$ band operation is almost not possible in a conventional fiber (dotted curve in figure 5). Higher wavelength ASE suppression in a $W$-fiber does enhance the $\mathrm{L}+$ band gain but the gain is sharply varying with the wavelength (dashed curve). If discrete filters at the end of the amplifier are used to flattened this gain, then the net gain of the amplifier cannot be better than $\sim 10 \mathrm{~dB}$ at all wavelengths. The solid curve shows the gain in the proposed bent $W$-fiber, where one may note that inherent gain flattening is achieved by a re-distribution of power along various signal channels. As a result, the higher wavelength gain is suppressed and the shorter wavelength gain is enhanced, leading to higher overall efficiency.

Increase in efficiency by inherent gain flattening can also be understood by studying the variation of population inversion along the length of the fiber and spectral variation of forward ASE power, for the above-mentioned three cases. These results are presented in Figures 6 and 7. The suppression of higher wavelength ASE (Figure 6) leads to significant increase in population inversion; this increase is further enhanced in the case of gain-flattened amplifier. One may note in Figure 7 that leakage loss in a $W$-fiber shifts the ASE spectrum to the shorter wavelengths, and the presence of bend-induced leakage loss flattens it. An advantage of the proposed scheme is that the gain-flattened amplification can be shifted to longer wavelengths (e.g. 1650-1700) nm also by a suitable choice of $\lambda_{c}$ and bend radius.

We have also carried out a detailed tolerance analysis for the fiber parameters, with respect to net gain and gain flattening in the proposed $\mathrm{L}+$ band TDFA. The analysis shows that it is the total leakage loss and its spectral variation, that primarily governs the gain flattening in the designed TDFA. We observed that any change in the loss spectrum (due to a variation in fiber parameters or in bend radius) can be well compensated by a re-optimization of fiber length, pump power and/or bend radius. Figure 8 shows the TDFA gain spectrum for different values of bend radius, keeping the fiber parameters and input signal powers to be the 
same as in Figure 4. $\mathrm{D}_{\mathrm{Rb}}$ represents the deviation in bend radius from its optimized value of 8 $\mathrm{cm}, \mathrm{P}_{\mathrm{p}}$ represents the pump power and $\mathrm{R}$ represents the gain ripple in each case. The gain flatness varies from $\pm 0.3 \mathrm{~dB}$ to $\pm 0.7 \mathrm{~dB}$ for $\mathrm{a} \pm 0.5 \mathrm{~cm}$

Figure 9 shows the results of tolerance analysis w.r.t core refractive index $n_{l} . \mathrm{D}_{\mathrm{n} 1}$ represents the deviation from optimum value, $\mathrm{R}$ represents the gain ripple and $\mathrm{P}_{\mathrm{p}}$ corresponds to the pump power in each case. The fiber length, ion concentration and bend radius have been re-optimized for each case. One may note that the gain spectra in the modified configuration does not deviate too much from the original curve (solid curve) and the gain flatness varies between $\pm 0.3 \mathrm{~dB}$ to $\pm 0.5 \mathrm{~dB}$. The other core parameters $\left(a\right.$ and $\left.n_{2}\right)$ have similar effect as of $n_{1}$. Any modification in these parameters basically leads to a shift in the cut-off wavelength, $\lambda_{c}$, and the effect can be compensated for in the same way as for the case of $n_{1}$. Figure 10 shows the corresponding analysis w.r.t separation between core and outer cladding, $b . \mathrm{D}_{\mathrm{b}}$ $(\mu \mathrm{m})$ corresponds to the deviation in the value of $b$. In this case also, the ion concentration, fiber length and bend radius have been re-optimized to ensure that the gain ripple (R), in each case, varies between \pm 0.3 to $\pm 0.4 \mathrm{~dB}$ only.

\section{Conclusions}

The paper reports a novel fluoride based inherently gain-flattened L+ TDFA design, based on the principle of shifting amplifier gain to shorter wavelengths, by suppressing higher wavelength (having higher gain coefficient) amplified spontaneous emission (ASE) using distributed leakage loss in a $W$-fiber. For achieving inherent gain-flattening, additional bendinduced leakage loss in the signal band has been utilized. Detailed modeling for amplifier characteristics has been carried out, including the effects of forward and backward ASE, pump depletion etc. $\mathrm{L}+$ band TDFA operation (20 dB gain) using $160 \mathrm{~mW}$ of pump power has been shown to be achievable over $32 \mathrm{~nm}$ bandwidth (1604-1636) $\mathrm{nm}$ with a gain ripple of $\pm 0.3 \mathrm{~dB}$. We have also shown that in this design, inherent gain flattening actually leads to an 
increase in overall efficiency. This is because in this design, inherent gain flattening is achieved by a suppression of gain at higher signal wavelengths and a simultaneous increase in gain at shorter signal wavelengths. This is in contrast to gain flattening by discrete filters, which is achieved by a suppression of gain at all wavelengths. A detailed tolerance analysis of gain and gain flattening w.r.t fiber parameters, as well as bend radius has been studied and it has been confirmed that in each case, the desired characteristics can be retained by a reoptimization of the amplifier parameters.

\section{Acknowledgements}

One of the authors (Charu Kakkar) would like to thank the French Embassy in India for awarding with a 'Sandwich' thesis scholarship. CK would also like to thank Basile Faure, University of Nice, for providing emission and absorption cross-sectional data for Thulium ions in fluoride-based fibers. 


\section{References}

1. H. Ono, M. Yamada, T. Kanamori, S. Sudo and Y. Ohishi, Journal of Lightwave Technology, 17, (1999), 490.

2. F. A. Flood, Proc. of Optocal fiber and Communications Conference (OFC-2000), (2000), 102.

3. A. J. G. Ellison, D. E. Goforth, B. N. Samson, J. D. Minelly, J. P. Trentelman, D. L. McEnroe and B. P. Tyndell, Proc. Optical Fiber Communication Conference (OFC 2001), 2, (2001), TuA2-1.

4. S. V. Chernikov, S. A. E. Lewis and J. R. Taylor, Proc. Optical Fiber Communication Conference (OFC 1999), (1999), WG6.

5. S. Aozasa, T. Sakamato, T. Kanamori, K. Hoshino, K. Kobayashi and M. Shimizu, IEEE Photonics Technology Letters, 12, (2000), 1331.

6. R. Percival, D. Szebesta, C. P. Seltzer, S. D. Perrin, S. T. Davey and M. Louka, IEEE Journal of Quantum Electronics, 31, (1995), 489.

7. T. Sakamoto, M. Shimizu, M. Yamada, T. Kanamori, Y. Ohishi, Y. Terunuma and Soichi Sudo, IEEE Photonics Technology Letters, 8, (1996), 349.

8. M. A. Arbore, Y. Zhou, H. Thiele, J. Bromage and L. Nelson, Proc. Optical Fiber Communication Conference, (OFC 2003), (2003), WK 2.

9. M. A. Arbore, Proc. Optical Fiber Communication Conference, (OFC 2005), Anaheim, USA, paper OFB 4, (2005).

10. D. Marcuse, Applied Optics, 21, (1982), 4208.

11. B. Pedersen, Optical and Quantum Electronics, 26, (1994), S273-S284.

12. K. Thyagarajan, S. Diggavi, A. Taneja and A. K. Ghatak, Applied Optics, 30, (1991), 3877. 


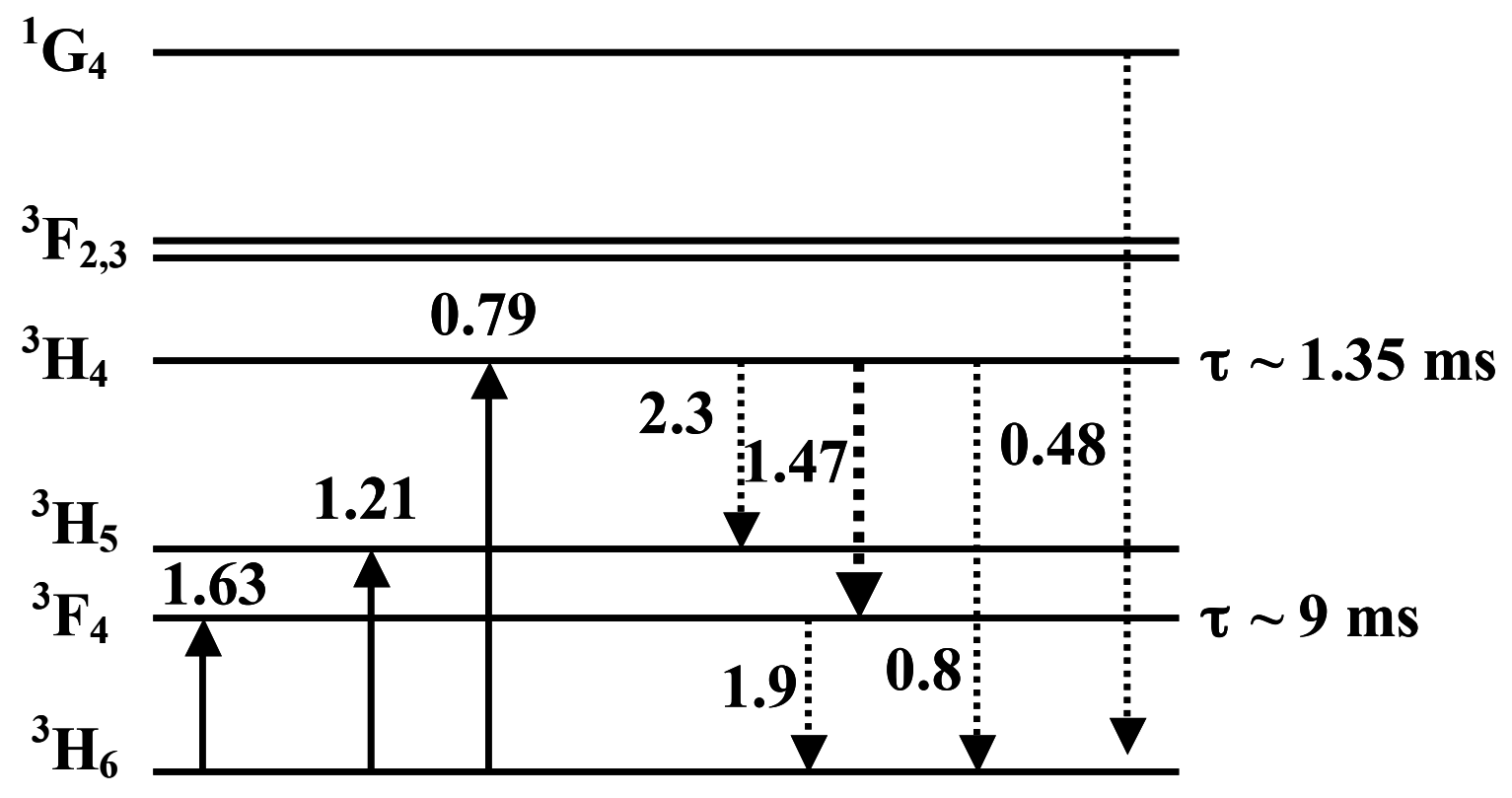

Fig. 1. Energy level diagram of $\mathrm{Tm}^{3+}$ ions in fluoride host; the numbers correspond to peak wavelength of transition, in microns. $\tau$ is the fluorescence lifetime of the level 


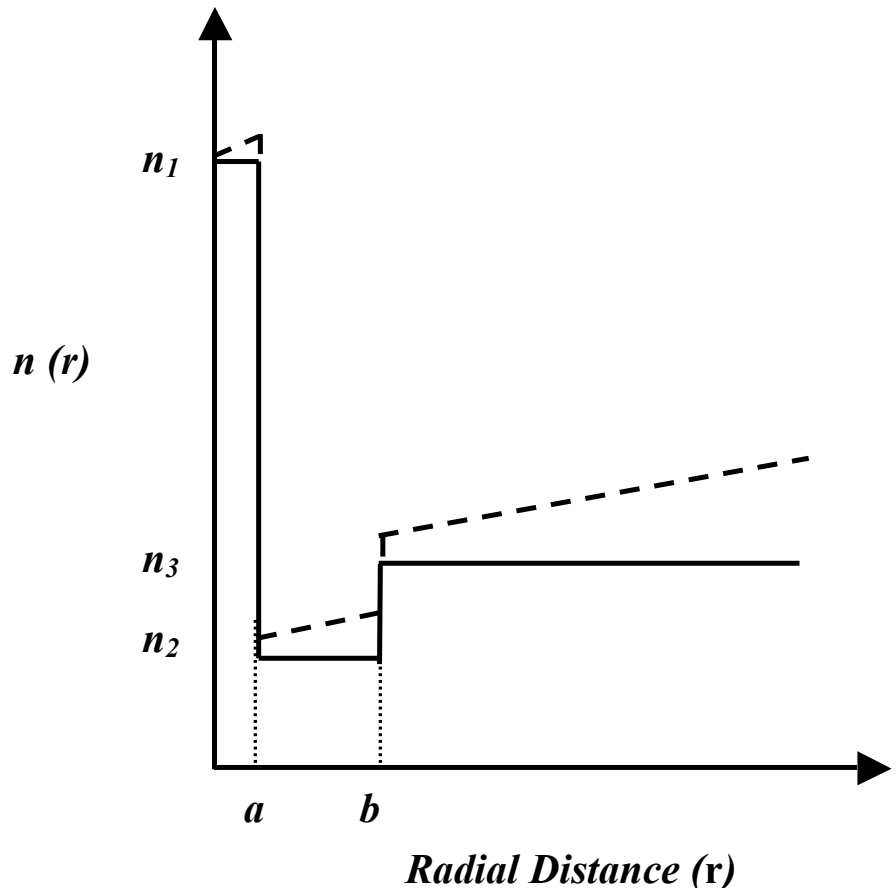

Fig. 2. Refractive index profile of a straight $W$-fiber (solid curve) and equivalent refractive index profile of a bent $W$-fiber (dashed curve) 


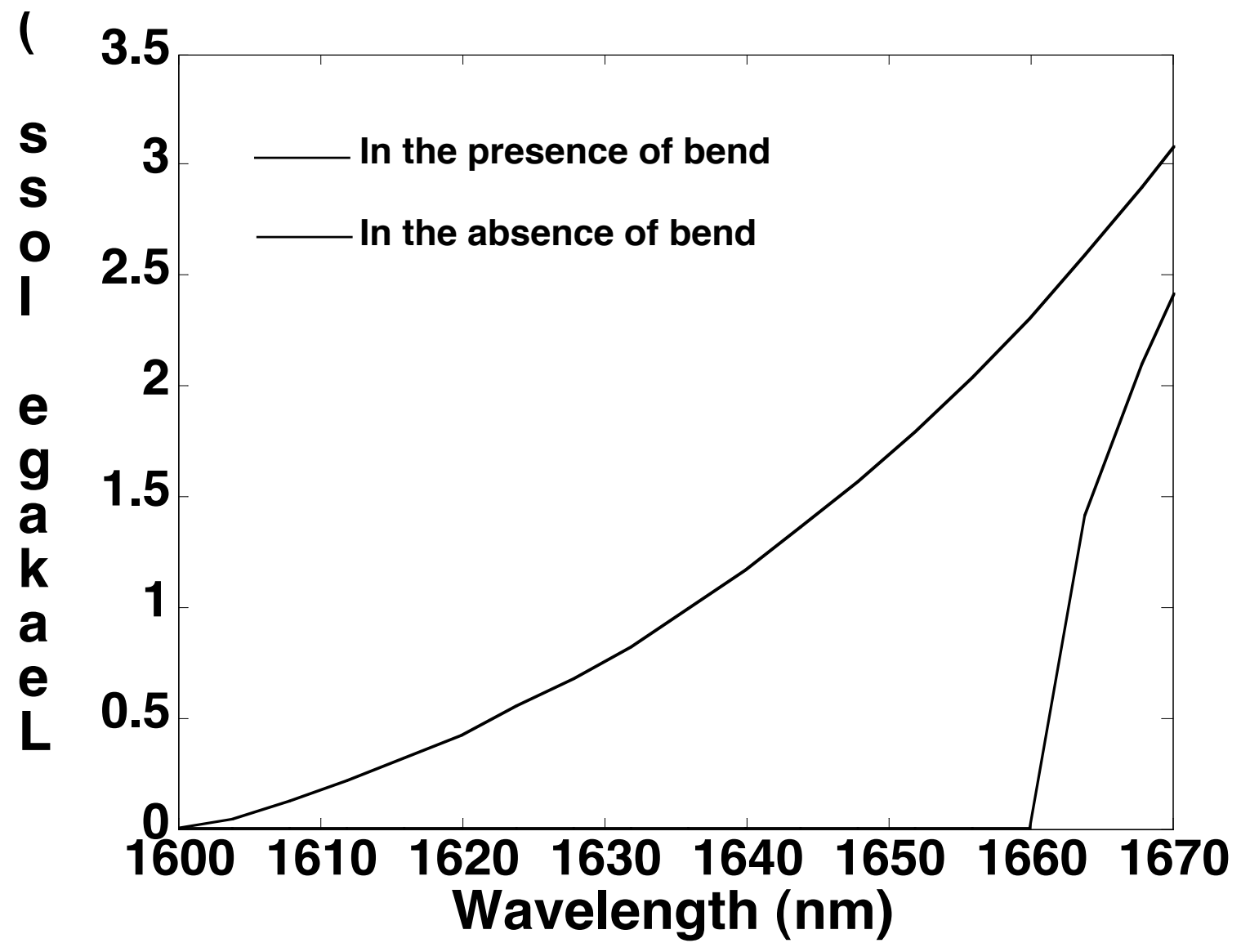

Fig. 3. Spectral variation of leakage loss in a $\mathrm{W}$-fiber, in the presence and absence of bend. Bend radius: $8 \mathrm{~cm}$ 


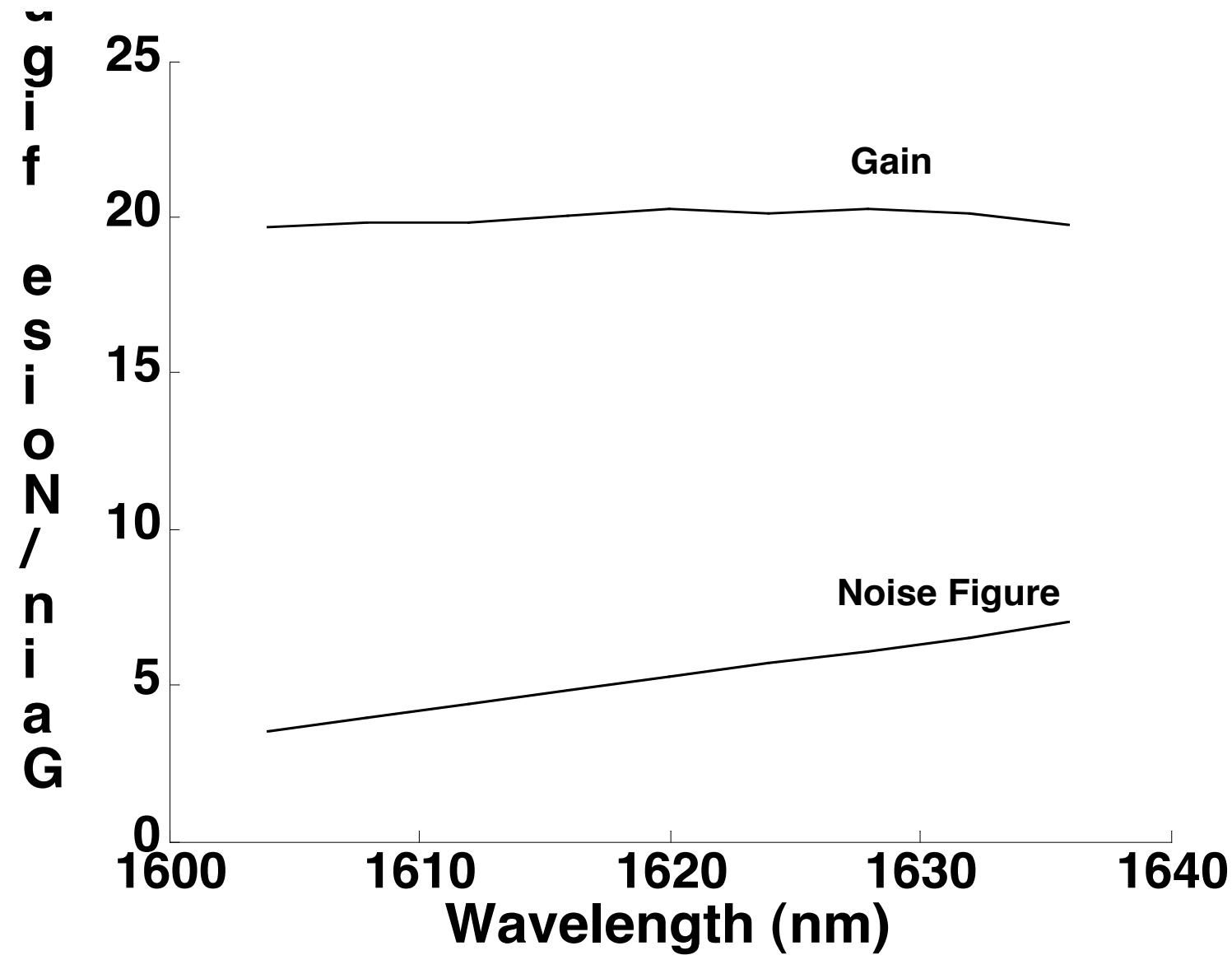

Fig. 4. Spectral variation of gain and noise figure in an $\mathrm{L}+$ band TDFA designed in an optimized, bent $\mathrm{W}$-fiber. $\mathrm{P}_{\mathrm{p}}=160 \mathrm{~mW}, \mathrm{~L}=32 \mathrm{~m}, \mathrm{P}_{\sin }=-10 \mathrm{dBm}$ 


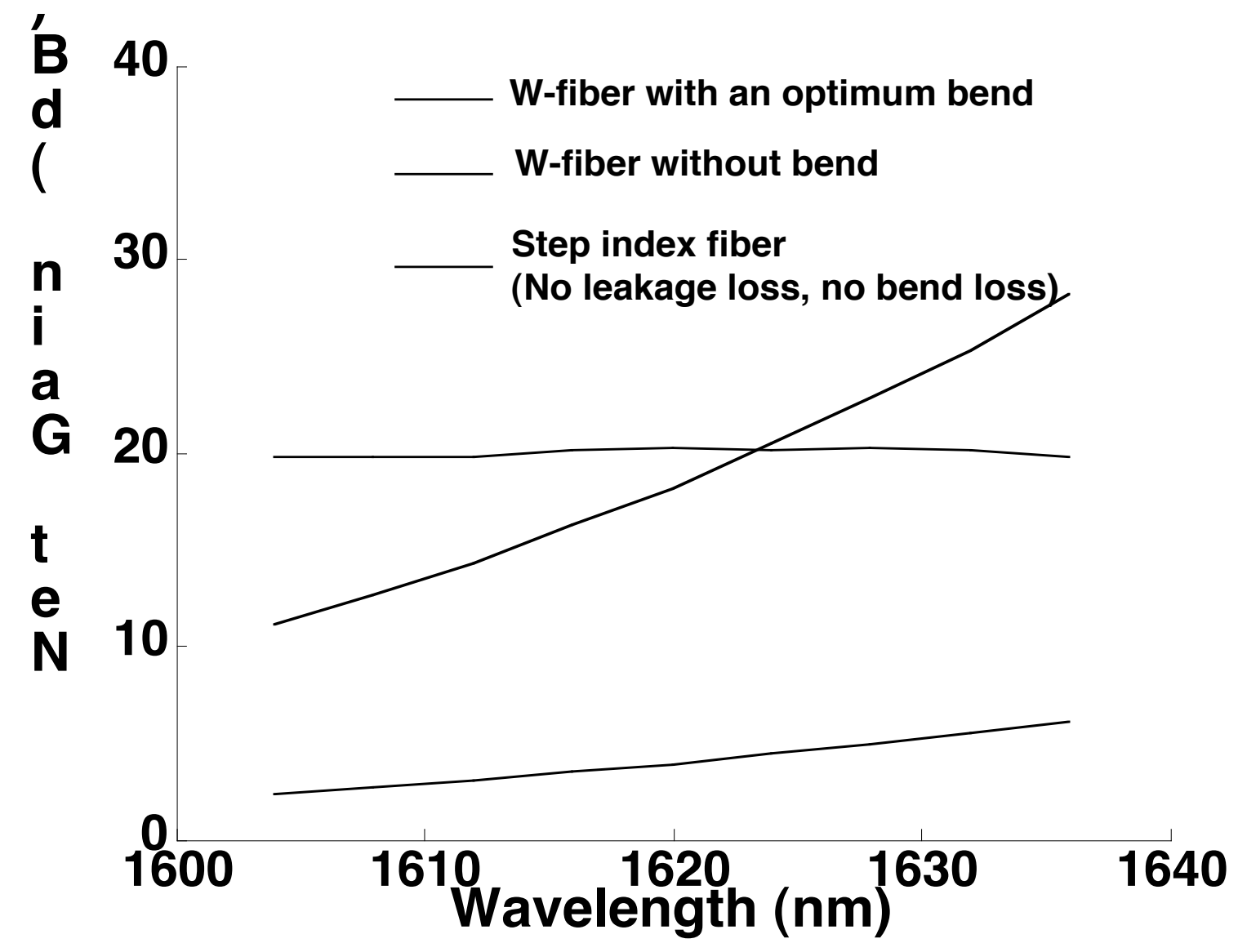

Fig. 5. Comparison of L-TDFA gain in the designed $\mathrm{W}$-fiber in the presence and absence of bend loss; corresponding gain variation in a step index fiber is also shown 


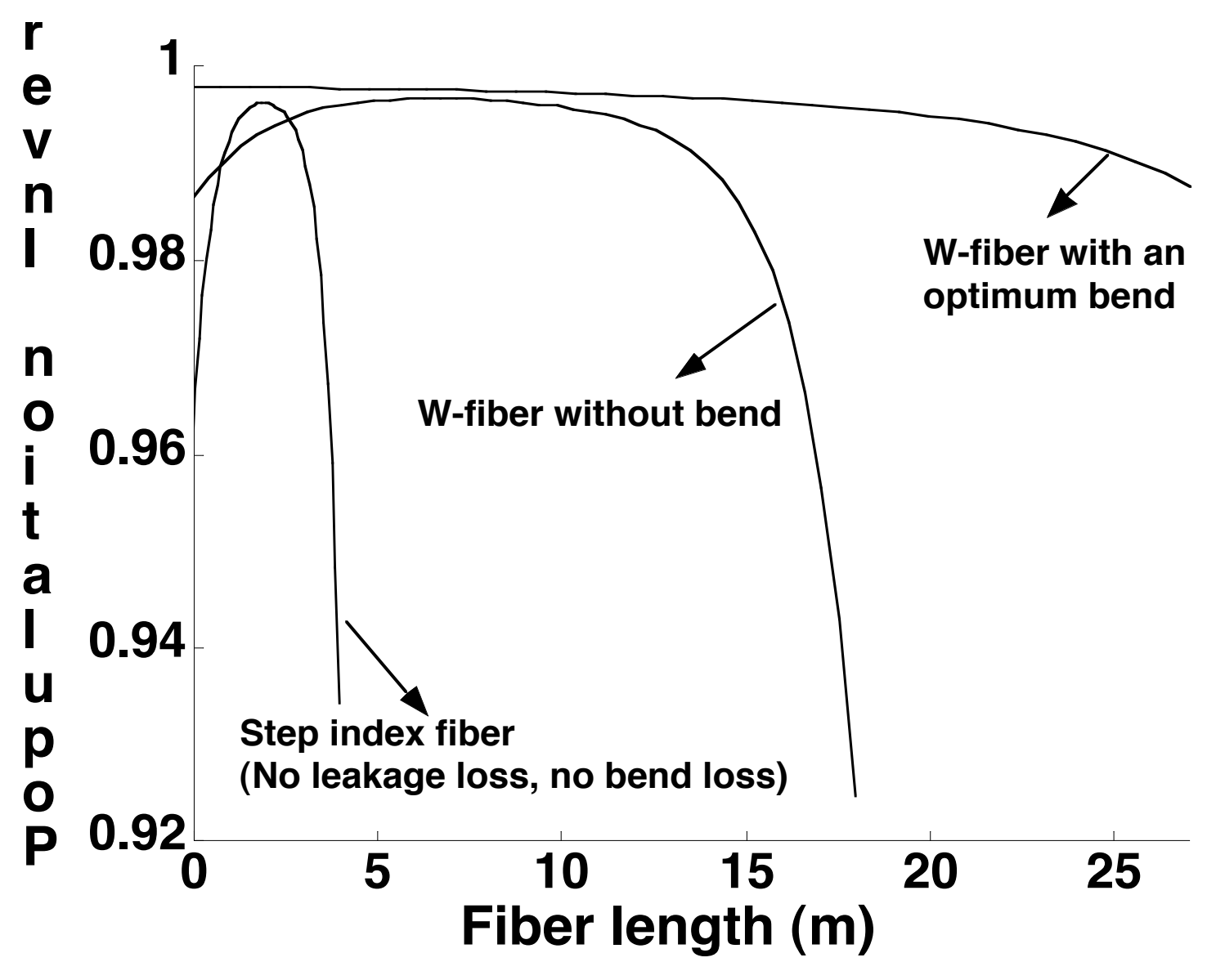

Fig. 6. Variation of population inversion along the length of fiber for the designed Wfiber, in the presence and absence of bend; corresponding variation in a step index fiher is also shown 


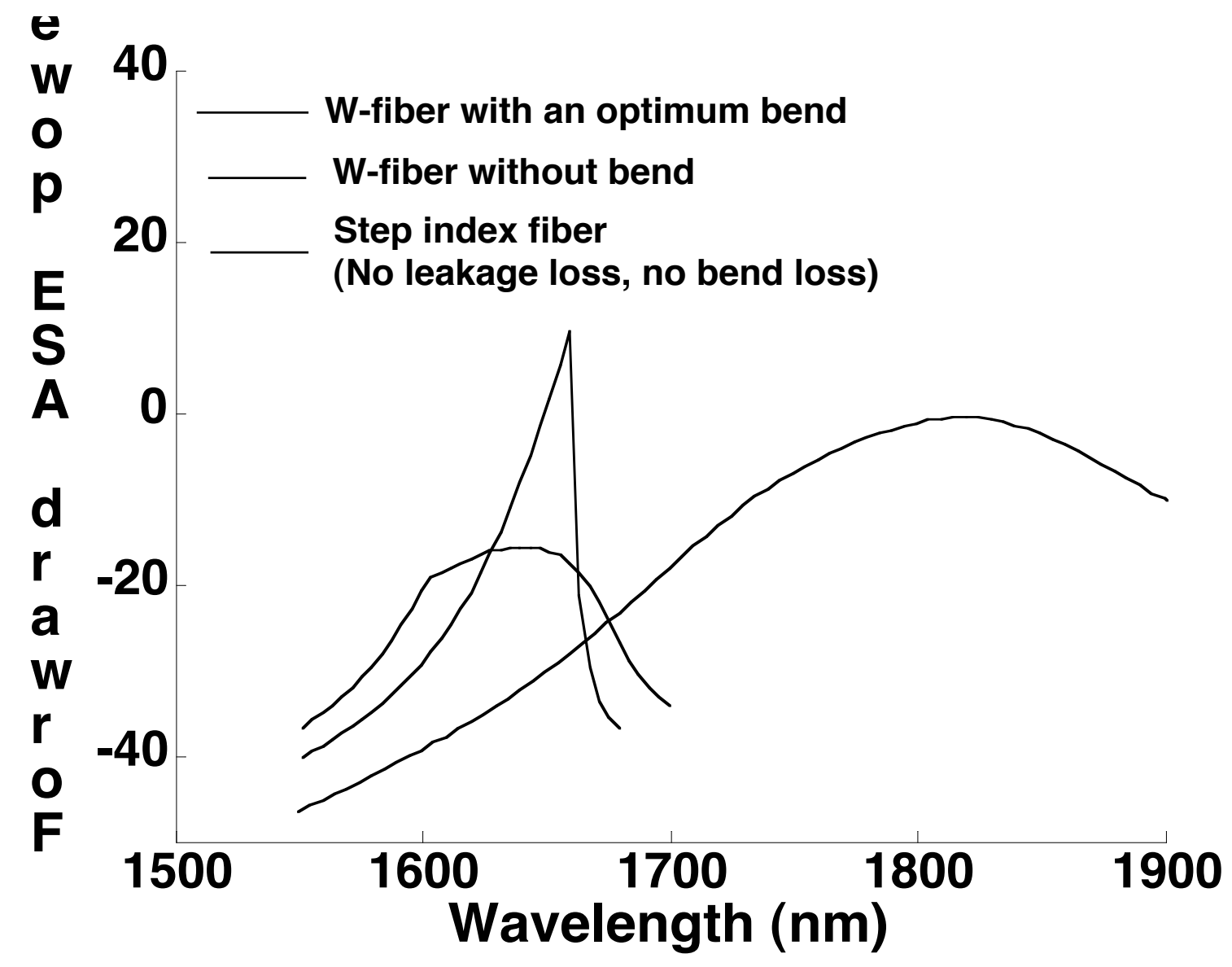

Fig. 7. Spectral variation of forward ASE power for the designed W-fiber, in the presence and absence of bend; corresponding variation in a step index fiber is also shown 


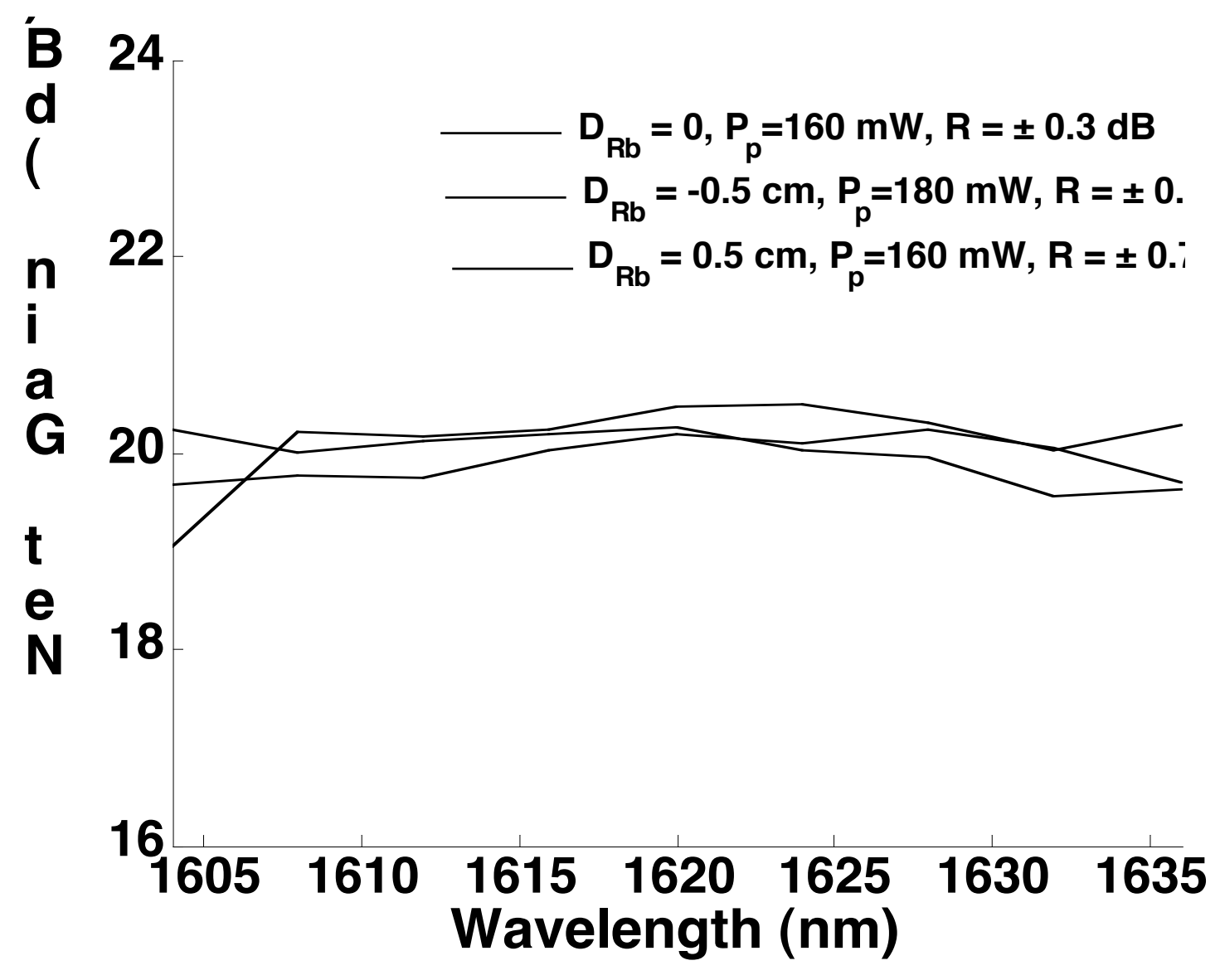

Fig. 8. Spectral variation of gain for different values of bend radius; $\mathrm{D}_{\mathrm{Rb}}$ corresponds to the deviation of bend radius from its optimized value of $8 \mathrm{~cm}$ and $\mathrm{R}$ is the gain ripple 


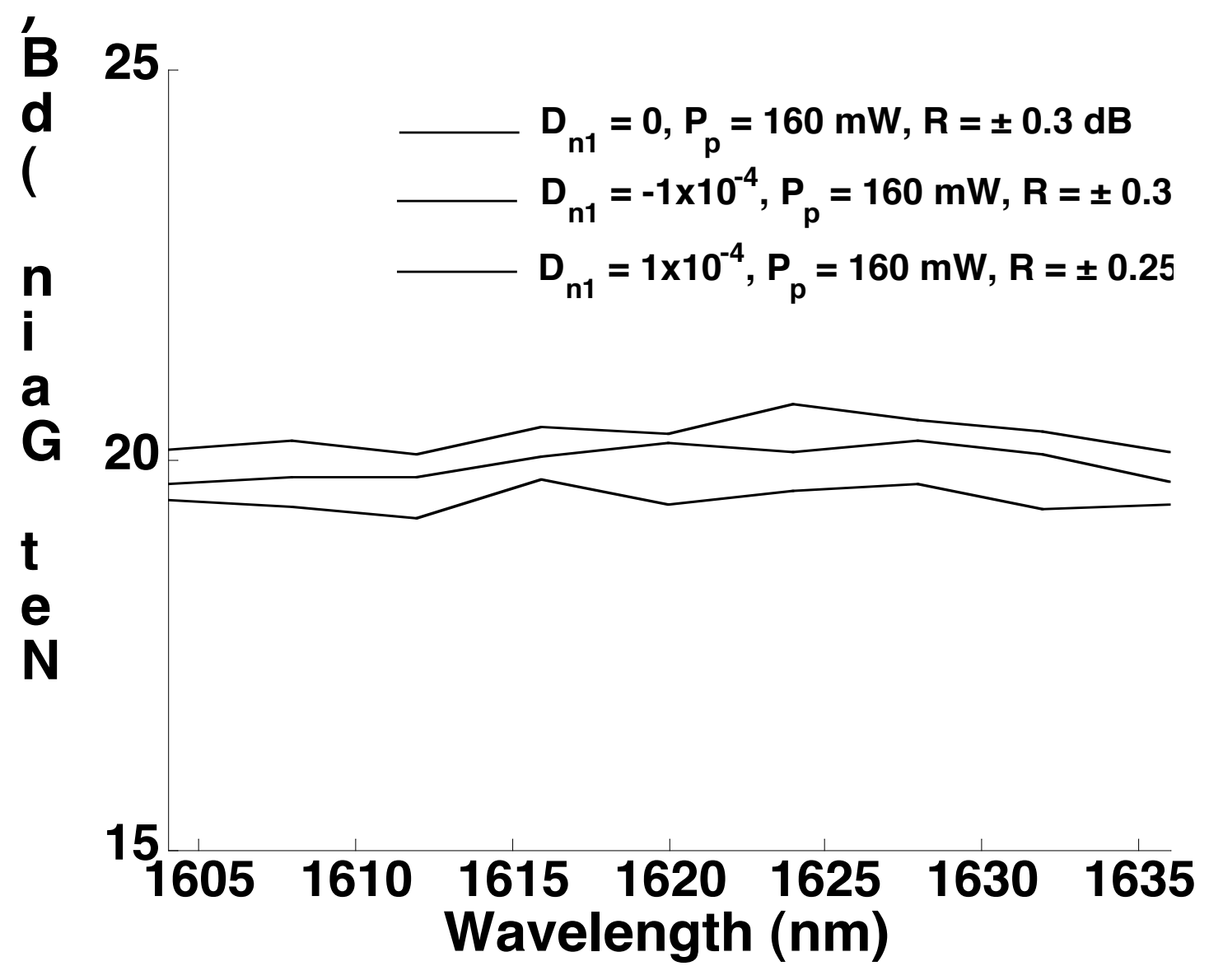

Fig. 9. Spectral variation of gain for different values of $n_{1} ; \mathrm{D}_{\mathrm{n} 1}$ corresponds to the deviation in $n_{l}$ from its optimized value and $\mathrm{R}$ is the gain ripple 


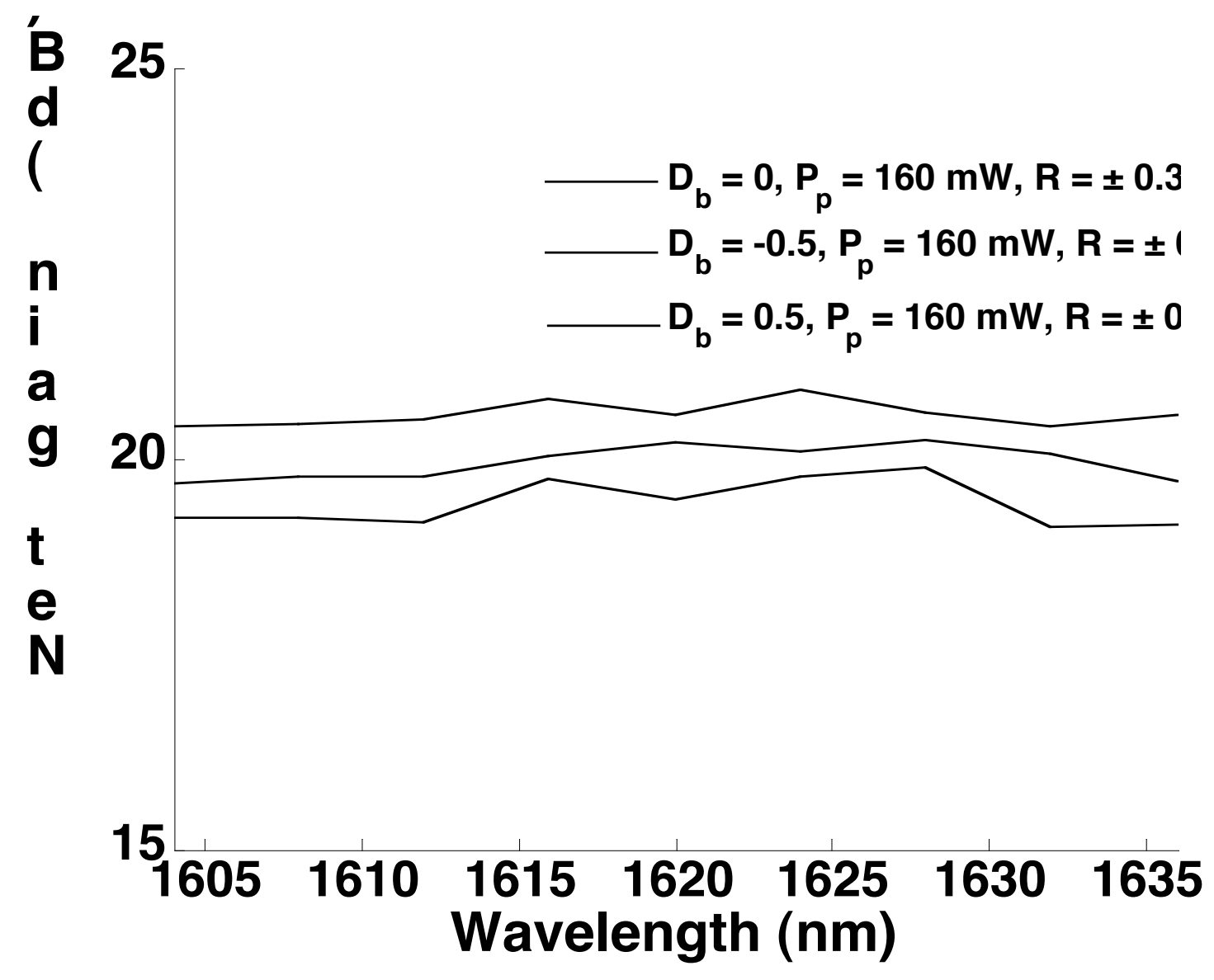

Fig. 10. Spectral variation of gain for different values of $b ; \mathrm{D}_{\mathrm{b}}(\mu \mathrm{m})$ corresponds to the deviation in $b$ from its optimized value and $\mathrm{R}$ is the gain ripple 